\title{
Therapeutic effect of the natural compounds baicalein and baicalin on autoimmune diseases
}

\author{
JIAN XU, JINLONG LIU, GUOLIN YUE, MINGQIANG SUN, JINLIANG LI, XIA XIU and ZHENZHONG GAO \\ Department of Pediatrics, Weifang Maternal and Child Health Hospital, Weifang, Shandong 261011, P.R. China
}

Received October 3, 2016; Accepted January 19, 2018

DOI: $10.3892 / \mathrm{mmr} .2018 .9054$

\begin{abstract}
A series of natural compounds have been implicated to be useful in regulating the pathogenesis of various autoimmune diseases. The present study demonstrated that the Scutellariae radix compounds baicalein and baicalin may serve as drugs for the treatment of autoimmune diseases, including rheumatoid arthritis and inflammatory bowel disease. Following the administration of baicalein and baicalin in vivo, T cell-mediated autoimmune diseases in the mouse model were profoundly ameliorated: In the collagen-induced arthritis model (CIA), the severity of the disease was reduced by baicalein and, consistently, baicalein was demonstrated to suppress $\mathrm{T}$ cell proliferation in CIA mice. In the dextran sodium sulfate (DSS)-induced colitis model, the disease was attenuated by baicalin, and baicalin promoted colon epithelial cell (CEC) proliferation in vitro. The present study further revealed that the mRNA expression of signal transducer and activator of transcription (STAT)3 and STAT4 in the tyrosine-protein kinase JAK-STAT signaling pathway in T cells was downregulated by baicalein, contributing to its regulation of $\mathrm{T}$ cell proliferation. However, in the DSS model, the STAT4 transcription in CECs, which are the target cells of activated $\mathrm{T}$ cells in the gut, was downregulated by baicalin, suggesting that baicalein and baicalin mediated similar STAT expression in different cell types in autoimmune diseases. In conclusion, the similarly structured compounds baicalein and baicalin selectively exhibited therapeutic effects on autoimmune diseases by regulating cell proliferation and STAT gene expression, albeit in different cell types.
\end{abstract}

\section{Introduction}

Millions of people are affected by T cell-mediated autoimmune diseases, including rheumatoid arthritis (RA), multiple sclerosis

Correspondence to: Dr Zhenzhong Gao, Department of Pediatrics, Weifang Maternal and Child Health Hospital, 407 Qingnian Road, Weifang, Shandong 261011, P.R. China

E-mail: ydukongjian@163.com

Key words: baicalein, baicalin, autoimmune diseases, tyrosine-protein kinase JAK-signal transducer and activator of transcription
(MS) and type 1 diabetes mellitus (1). Although autoimmune diseases occur within different tissues, disease-associated autoimmune $\mathrm{T}$ cells are frequently autoreactive and proliferate in the pathological state. By contrast, the cells in the lesion tissue targeted by T cells are injured by apoptosis or a reduced proliferative rate (2).

Previously, there have been reports about the role of the Scutellariae radix compounds baicalein and baicalin $(3,4)$. For example, baicalein, baicalin and wogonin have been demonstrated to serve roles in bladder cancer cell lines including inhibition of cell proliferation in a dose-dependent manner. Of the aforementioned compounds, baicalin exhibited the greatest antiproliferative activity on human bladder cancer cell lines (KU-1 and EJ-1) and a murine bladder cancer cell line (MBT-2) $(5,6)$. In addition, these compounds have been reported to exhibit strong antioxidant and free radical-scavenging activity (7). It was reported that baicalein was able to significantly attenuate the clinical severity of experimental autoimmune encephalomyelitis (EAE), an animal model of MS (8). The inhibited migration of autoimmune $\mathrm{T}$ cells into the central nervous system (CNS) caused by treatment with baicalein may be attributed to the reduced activation of microglia, which was indicated previously by suppressed phagocytosis, and decreased production of proinflammatory cytokines and chemokines in the CNS $(9,10)$. Baicalein additionally selectively induces apoptosis in activated lymphocytes and ameliorates concanavalin A-induced hepatitis in mice (11). Although baicalein and baicalin have similar chemical structure, baicalin exhibited slightly different effects in autoimmune diseases via various mechanisms. For instance, baicalin exhibited protective effects on myelin in rats with EAE, although the anti-inflammatory activity of baicalin is due to the binding of chemokines $(12,13)$. The present study tested the effect of baicalein and baicalin in other autoimmune diseases, including an RA model and a colitis model, and compared the different mechanisms of the autoimmune disorders. The results of the present study may provide a basis for the treatment of autoimmune diseases using these natural compounds.

\section{Materials and methods}

Chemicals. Baicalein and baicalin (Fig. 1A) were purchased from Sigma-Aldrich (Merck KGaA, Darmstadt, Germany). For the in vitro study, the drugs were used at a concentration of $100 \mu \mathrm{M}$ baicalein and $200 \mu \mathrm{M}$ baicalin. The compounds 
were added $1 \mathrm{~h}$ prior to cell activation. For the in vivo study, baicalein and baicalin were both used at a dose of $20 \mathrm{mg} / \mathrm{kg}$.

Ethics statement. All animal procedures were performed in strict accordance with the recommendations in the Guide for the Care and Use of the Animal Biosafety Level 3 Laboratory (of Wuhan University (Wuhan, China). The protocol was approved by the Committee on the Ethics of Animal Experiments of Wuhan University (permit no. SCXK 2008-0004).

Collagen-induced arthritis (CIA) model. A total of $50 \mathrm{C} 57 \mathrm{BL} / 6 \mathrm{~J}$ male mice (6-8 weeks of age; weight, $25 \mathrm{~g}$ ) were obtained from the Animal Biosafety Level 3 Laboratory of Wuhan University. The mice were housed under standard laboratory conditions (a 12-h light/dark cycle with an average room temperature of $23^{\circ} \mathrm{C}$ and a relative humidity of $55 \%$, with food and water available ad libitum). Mice received a subcutaneous injection of $0.3 \mathrm{ml}$ collagen (Sigma-Aldrich; Merck $\mathrm{KGaA}$ ) at the base of the tail. The vehicle group was treated with PBS. Affected joints were counted daily for 35 days, mice were sacrificed at the end of the treatment with baicalein/baicalin and the synovial fluids (SF) were processed for ELISA analysis.

DSS induced colitis. Colitis was induced in mice with $2.5 \%$ w/v DSS (MP Biomedicals, LLC, Santa Ana, CA, USA) provided ad libitum for 9 days. DSS induces mucosal damage in the colon, resulting in an influx of commensal flora to the submucosal layers; this induces an immune response that closely parallels human colitis. Control groups received tap water only, DSS group received 2.5\% DSS, DSS + baicalein group received $2.5 \%$ DSS and baicalein treatment, and DSS + baicalin group was treated with $2.5 \%$ DSS and baicalin.

Reverse transcription-quantitative polymerase chain reaction (RT-qPCR). Cells The cells are colon epithelial cells isolated from DSS mice and the lymphocytes isolated from the synovial fluid of CIA mice. For isolation of colon epithelial cells, the entire colon was dissected out and cut longitudinally. The feces were removed and the lumen was cleaned with PBS. The colon was subsequently cut into 1-cm-long pieces, transferred into a $50 \mathrm{ml}$ conical flask containing PBS and stirred vigorously at $37^{\circ} \mathrm{C}$ for $30 \mathrm{~min}$. The tissue was subsequently strained through a tea strainer and the debris was washed in PBS to isolate any residual epithelial cells. The collected PBS solution was centrifuged at $1,000 \mathrm{x} \mathrm{g}$ for $5 \mathrm{~min}$ at room temperature. The cells were then suspended in $1 \mathrm{ml}$ PBS, added to a Percoll gradient (40 and 25\% Percoll solutions, GE Healthcare, Chicago, IL, USA), and subjected to density gradient centrifugation for 25-30 min at 1,000 $\mathrm{x} g$ at room temperature. Subsequently, the cells at the interface (colon epithelial cells) were collected, washed twice with PBS and used for isolation of RNA. CD4 ${ }^{+} \mathrm{T}$ cells were sorted by fluorescence-activated cell sorting from the SF of CIA mice. FACS for $\mathrm{CD}^{+} \mathrm{T}$ cells was performed using the BD FACS Aria flow cytometer (BD Biosciences, San Jose, CA, USA) by gating on $\mathrm{CD}^{+}$cells after Fc block. Cells were washed once with staining buffer [Pharmingen Stain Buffer containing bovine serum albumin (BSA) proteins; BD Biosciences; cat. no. 554657]. The supernatant was discarded and cells were resuspend in the staining buffer at a density up to $50 \times 10^{6}$ cells $/ \mathrm{ml}$ to maximize the efficiency of staining. For lymphocytes expressing high levels of Fc receptor (FcR), a monoclonal antibody that binds to FcgR (Purified Rat Anti-Mouse CD16/CD32; Mouse BD Fc Block $^{\mathrm{TM}}$; cat. no. 553142; BD Biosciences) was used to bind the receptor on ice for 10-15 min. Subsequently, CD4 mAb (FITC Rat Anti-Mouse CD4; cat. no. 553046; BD Biosciences) was used to detect the desired cell population by incubation for 20-30 min on ice in the dark, followed by two washes with Pharmingen Stain Buffer containing BSA proteins. Finally, the cell sorting analysis was performed at $4^{\circ} \mathrm{C}$. The data were analyzed with the FlowJo software version 6.3.2 (Tree Star, Inc., Ashland, OR, USA). Cells were treated with baicalein/baicalin in vitro and the culture period was $8 \mathrm{~h}$. Total RNA was extracted from the cells using the RNeasy mini kit (Qiagen China Co., Ltd., Shanghai, China), followed by cDNA synthesis using the Superscript III first strand synthesis kit (Invitrogen; Thermo Fisher Scientific, Inc., Waltham, MA, USA) at $25^{\circ} \mathrm{C}$ for $10 \mathrm{~min}, 50^{\circ} \mathrm{C}$ for $30 \mathrm{~min}$ and $85^{\circ} \mathrm{C}$ for $5 \mathrm{~min}$. qPCR was performed on a Bio-Rad amplifier using the Bio-Rad real time PCR mix, including SYBR Green dye (both Bio-Rad Laboratories, Inc., Hercules, CA, USA). The following thermocycling conditions were used for the PCR: $50^{\circ} \mathrm{C}$ for $2 \mathrm{~min}$, $10 \mathrm{~min}$ at $95^{\circ} \mathrm{C} ; 40$ cycles of $95^{\circ} \mathrm{C}$ for $15 \mathrm{sec}$ and $60^{\circ} \mathrm{C}$ for $1 \mathrm{~min}$. Data were analyzed using the $\mathrm{Cq}$ value normalized to the endogenous reference gene GAPDH (14). The following primers were used for the PCR: Ki-67 (forward: 5'-ACCGTG GAGTAGTTTATCTGGG-3'; reverse: 5'-TGTTTCCAGTCC GCTTACTTCT-3'); STAT3 (forward: 5'-CAATACCATTGA CCTGCCGAT-3'; reverse: 5'-GAGCGACTCAAACTGCCC T-3'); STAT4 (5'-forward: TGGCAACAATTCTGCTTCAAA AC-3'; reverse: 5'-GAGGTCCCTGGATAGGCATGT-3'); STAT6 (5'-forward: CTCTGTGGGGCCTAATTTCCA-3'; reverse: 5'-CATCTGAACCGACCAGGAACT-3'); GAPDH (5'-forward: AGGTCGGTGTGAACGGATTTG-3'; forward: 5'-TGTAGACCATGTAGTTGAGGTCA-3').

ELISA analysis. Cytokine production was measured using commercial kit for interleukin (IL)-2 (cat. no. DY402), interferon (IFN)- $\gamma$ (cat. no. DY485), tumor necrosis factor (TNF)- $\alpha$ (cat. no. DY410; all R\&D Systems, Inc., Minneapolis, MN, USA), IL-6 (cat. no. 550950) and IL-17 (cat. no. 555067; both BD Pharmingen; BD Biosciences), according to the manufacturers' protocols.

MTT assay. Cells were incubated with MTT reagent $(5 \mu \mathrm{g} / \mathrm{ml}$ final concentration) at $37^{\circ} \mathrm{C}$ for $4 \mathrm{~h}$. Formazan was solubilized by adding $100 \mu \mathrm{l}$ DMSO into each well. The extent of formazan production was determined by an ELISA reader at a wavelength of $550 \mathrm{~nm}$, while $630 \mathrm{~nm}$ served as the reference wavelength. The results were calculated according to the manufacturer's instructions (Vybrant ${ }^{\mathrm{TM}}$ MTT Cell Proliferation Assay kit; Thermo Fisher Scientific, Inc.).

Histology. The colon was washed with cold PBS, measured and weighed. It was Swiss-rolled and sections were stained with hematoxylin and eosin as described previously (15). A histopathological score was generated in a blinded fashion using a widely used grading tool (16) examining crypts, 
epithelia, goblet cells, cellular infiltration and edema. Scores of 0-1 reflect normal morphology, and 2-4, 5-7 and 8-10 represent mild, moderate, and severe colitis, respectively.

Statistical analysis. The data were analyzed using SPSS 17.0 software (SPSS, Inc., Chicago, IL, USA) to evaluate the differences between the groups. The data are reported as the mean \pm standard deviation. $\mathrm{P}<0.05$ was considered to indicate a statistically significant difference. One-way analysis of variance with the Student-Newman-Keuls post hoc test was used to calculate the differences between the multiple comparison groups. The Mann-Whitney U test was used to analyze the differences between two groups.

\section{Results}

Baicalein attenuates mouse CIA. Baicalein and baicalin administration exhibited protective activity in two animal models in vivo. Firstly, the effect on the mouse CIA model was investigated. Mice developed stable arthritis symptoms on day 10 and the severity of the disease in the vehicle-treated group worsened continuously with time. The baicalein-treated group had a lower arthritis score and fewer affected joints compared with the vehicle group during the entire course of treatment, while baicalin exerted no significant effect on the CIA model (Fig. 1B and C). This result was confirmed by measuring cytokine production in the SF. Baicalein was able to suppress Th1-type cytokine production, including IFN- $\gamma$, TNF- $\alpha$ and IL-2, in addition to the Th17-type cytokine IL-17, suggesting that it may inhibit $\mathrm{T}$ cell function in vivo (Fig. 1D).

Baicalein regulates CIA mouse T cell proliferation via signal transducer and activator of transcription (STAT) expression in tyrosine-protein kinase JAK (JAK)/STAT signaling. Furthermore, the role of baicalein in $\mathrm{T}$ cell proliferation was investigated. Treatment with $100 \mathrm{nM}$ baicalein in vitro significantly inhibited the proliferation of $\mathrm{CD} 4^{+} \mathrm{T}$ cells sorted from the SF of CIA mice upon anti-CD3 Ab activation, demonstrated via the MTT assay, and supported by the Ki67 expression analysis using qPCR (Fig. 2A and B). To elucidate the primary mechanism underlying the role of baicalein in proliferation, JAK/STAT pathway gene expression was assessed and the cells were treated with baicalein in vitro. As presented in Fig. 2C, baicalein significantly reduced the expression of STAT3 and STAT4, although it did not exert the same effect on STAT6 expression in infiltrated SF T cells (Fig. 2C), suggesting that baicalein regulated joint inflammatory signaling by suppressing STAT3/STAT4 expression in the JAK/STAT pathway.

Baicalin ameliorates DSS-induced colitis. The efficacy of baicalein and baicalin was investigated in another type of autoimmune disease. A DSS-induced colitis model in mice was produced via treatment with 3\% DSS. The body weight of the baicalin-treated group was increased compared with the vehicle-treated group at the peak of the disease, which was consistent with the colon length and histology scores, while baicalein exerted no effect on the DSS model (Fig. 3A-C). Similarly, the serum IL-2, IFN- $\gamma$ and IL-17 levels were reduced by baicalin (Fig. 3D).<smiles>O=c1cc(-c2ccccc2)oc2cc(O)c(O)c(O)c12</smiles>

Baicalein

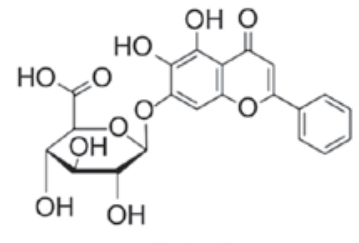

Baicalin
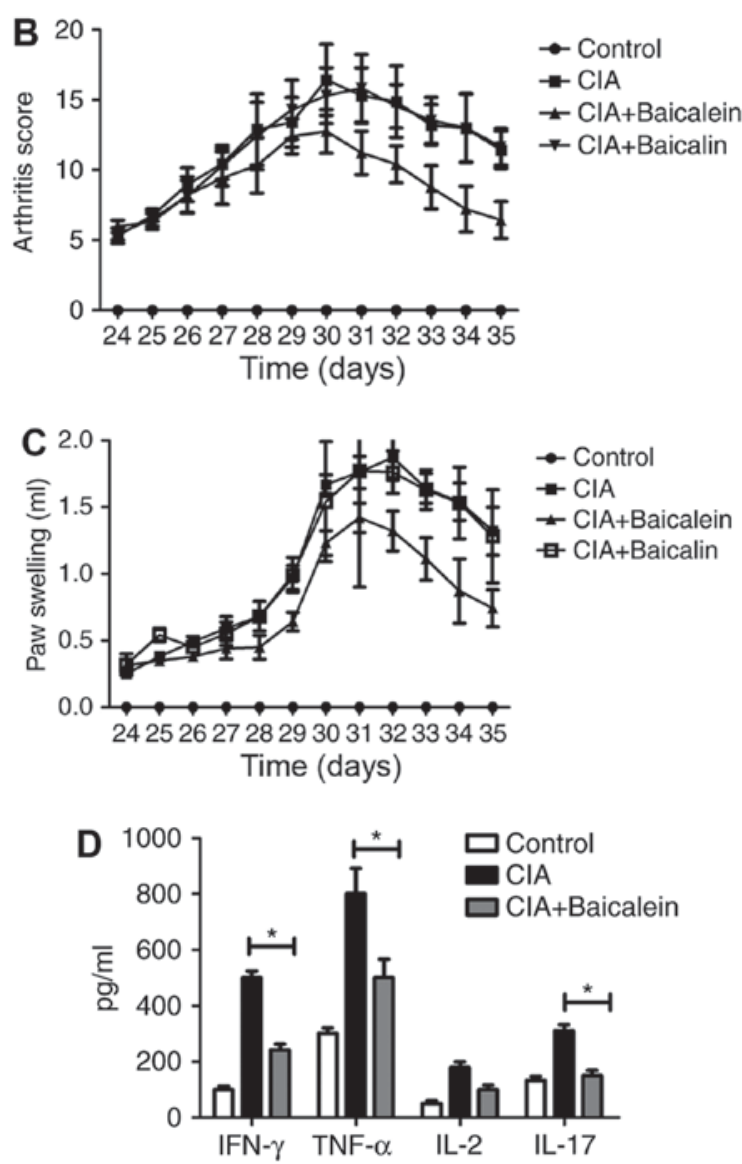

Figure 1. Effect of baicalein and baicalin on the CIA model. (A) The chemical structures of the drugs baicalein and baicalin. (B) Arthritis scores in the CIA model following treatment with baicalein and baicalin. (C) Paw swelling of CIA model mice following treatment with baicalein and baicalin. (D) Cytokine production in the synovial fluid of each group was measured using ELISA analysis. The data are presented as the mean \pm standard deviation. ${ }^{*} \mathrm{P}<0.05$. CIA, collagen-induced arthritis; IFN- $\gamma$, interferon- $\gamma$; TNF- $\alpha$; tumor necrosis factor- $\alpha$; IL, interleukin.

Baicalin regulated epithelial cell proliferation in DSS-induced colitis via STAT expression in JAK/STAT signaling. To determine the effect of baicalin on epithelial proliferation, colon epithelial cells (CECs) were isolated and treated with baicalin in vitro. Baicalin was able to promote the proliferation of CECs as measured by CCK8 staining, suggesting that it was able to repair the epithelial barrier and promote mucosal wound healing in the disease state (Fig. 4A). This was consistent with the proliferation marker Ki67 analysis using qPCR (Fig. 4B). In order to elucidate the possible mechanism underlying the effect of baicalin on CEC proliferation, the JAK-STAT signaling pathway was selected for analysis and the mRNA expression of STAT subtype genes was assessed by qPCR. It was demonstrated that baicalin may reduce the expression of the STAT4 gene locus, while 

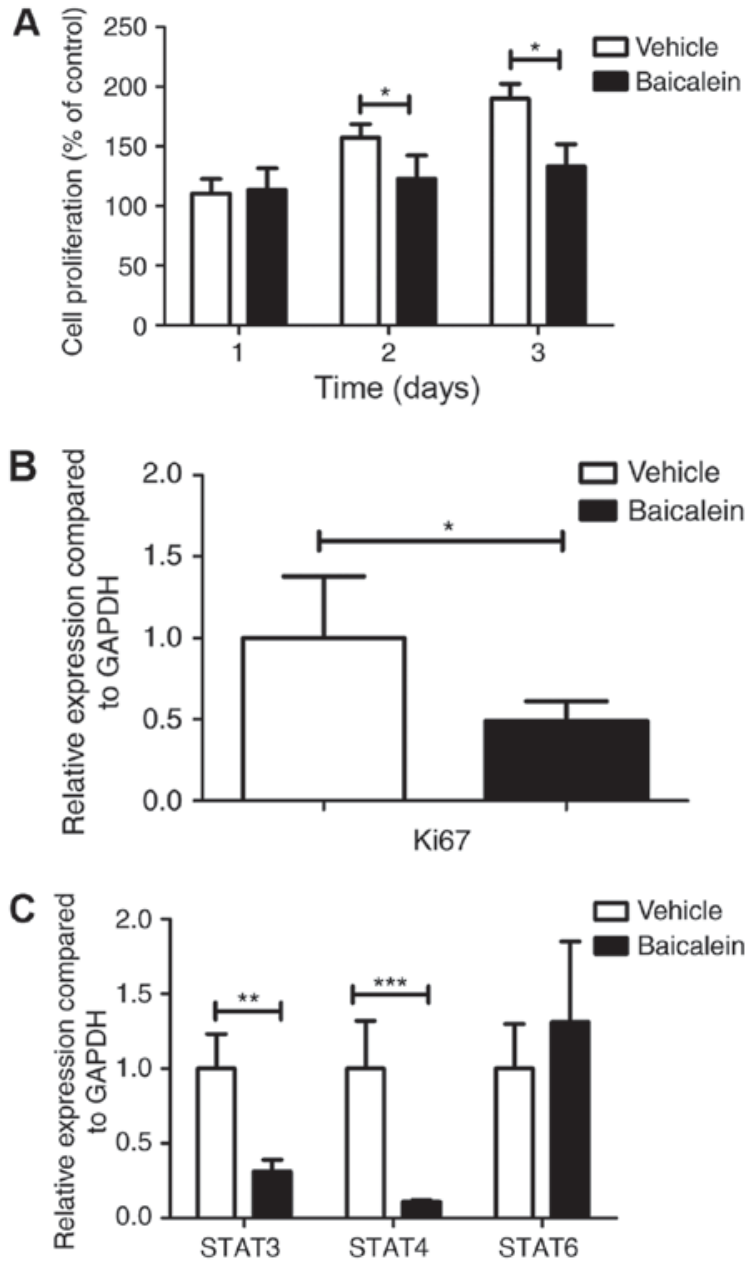

Figure 2. Baicalein regulates $\mathrm{T}$ cell proliferation and STAT expression. (A) MTT assay of proliferation of $\mathrm{CD}^{+} \mathrm{T}$ cells sorted from the SF of CIA mice upon anti-CD3 Ab activation by treatment with baicalein in vitro. (B) The Ki67 mRNA expression of $\mathrm{CD}^{+} \mathrm{T}$ cells sorted from the SF of CIA mice upon anti-CD3 Ab activation by treatment with baicalein in vitro. (C) The STAT3/4/6 mRNA expression of $\mathrm{CD}^{+}{ }^{+} \mathrm{T}$ cells sorted from the SF of CIA mice upon anti-CD3 Ab activation by treatment with baicalein in vitro. The data are presented as the mean \pm standard deviation. ${ }^{*} \mathrm{P}<0.05,{ }^{* *} \mathrm{P}<0.01$, ${ }^{* * * *} \mathrm{P}<0.001$. Ab, antibody; STAT, signal transducer and activator of transcription; SF, synovial fluid; CIA, collagen-induced arthritis.

having no significant effect on STAT3/STAT6 (Fig. 4C), suggesting that baicalin mediated CEC proliferation through the JAK-STAT signaling pathway.

\section{Discussion}

The results of the present study demonstrated that the components of Scutellariae radix (termed Huang-Qin in Chinese), baicalein and baicalin, regulate $\mathrm{T}$ cell and epithelial cell function to exert a therapeutic effect on autoimmune disease. The distinct biological activities of baicalein and baicalin were assessed in diverse autoimmune diseases. Baicalein ameliorated the severity of disease in the CIA model, while baicalin attenuated DSS-induced colitis; the in vitro study demonstrated that the two compounds mediated cell activation via the JAK-STAT pathway and cell proliferation. It has been reported that baicalein and baicalin exert multiple physiological activities. Baicalin ameliorates camptothecin-induced intestinal toxicity
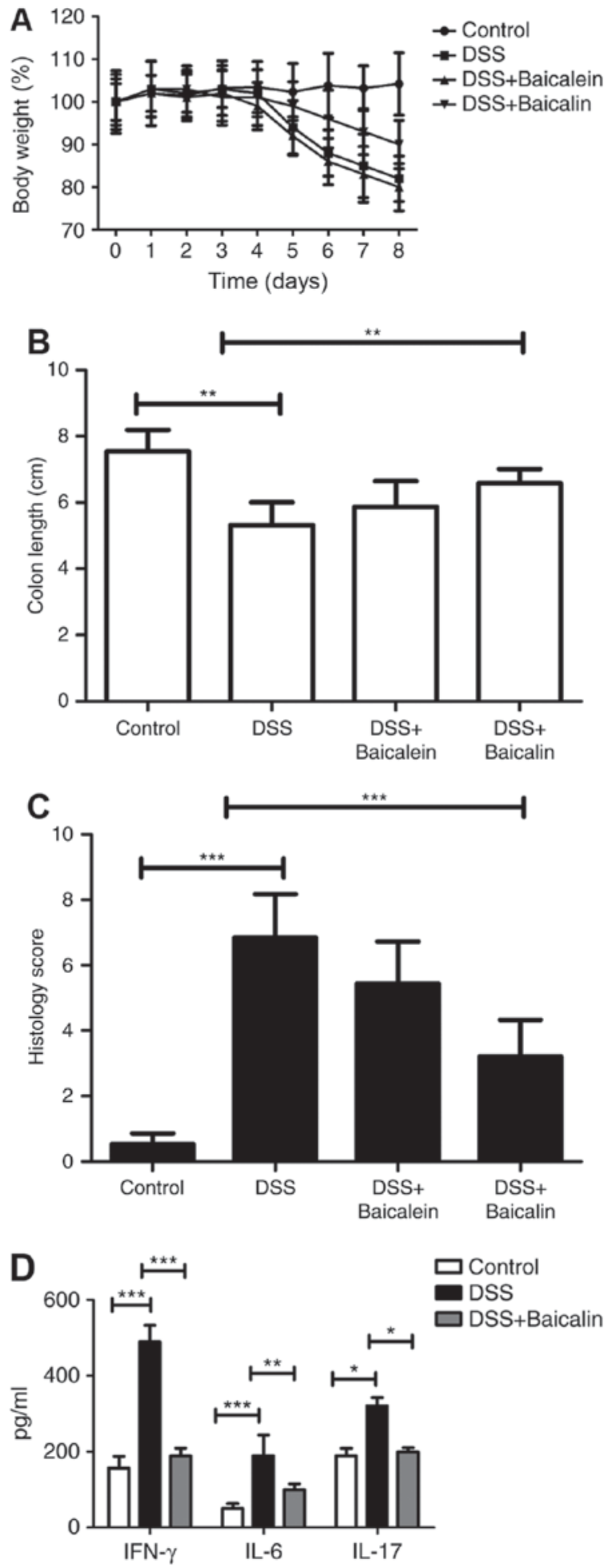

Figure 3. Effect of baicalein and baicalin on DSS-induced colitis model mice. (A) Body weight loss of DSS model mice following treatment with baicalein and baicalin. (B) Body weight loss of DSS model mice following treatment with baicalein and baicalin. (C) Histology scores of DSS model mice following treatment with baicalein and baicalin. (D) Cytokine production in the serum of each group was measured by ELISA. ${ }^{*} \mathrm{P}<0.05,{ }^{* *} \mathrm{P}<0.01$, ${ }^{* * * *} \mathrm{P}<0.001$. DSS, dextran sodium sulfate; IFN- $\gamma$, interferon- $\gamma$; IL, interleukin.

in rats (17). In vitro, baicalin exhibited a protective role in renal cell injury (18). It has additionally been reported that baicalein and baicalin are potent inhibitors of reverse transcriptase, and that they suppress the human T-cell leukemia virus and promote 

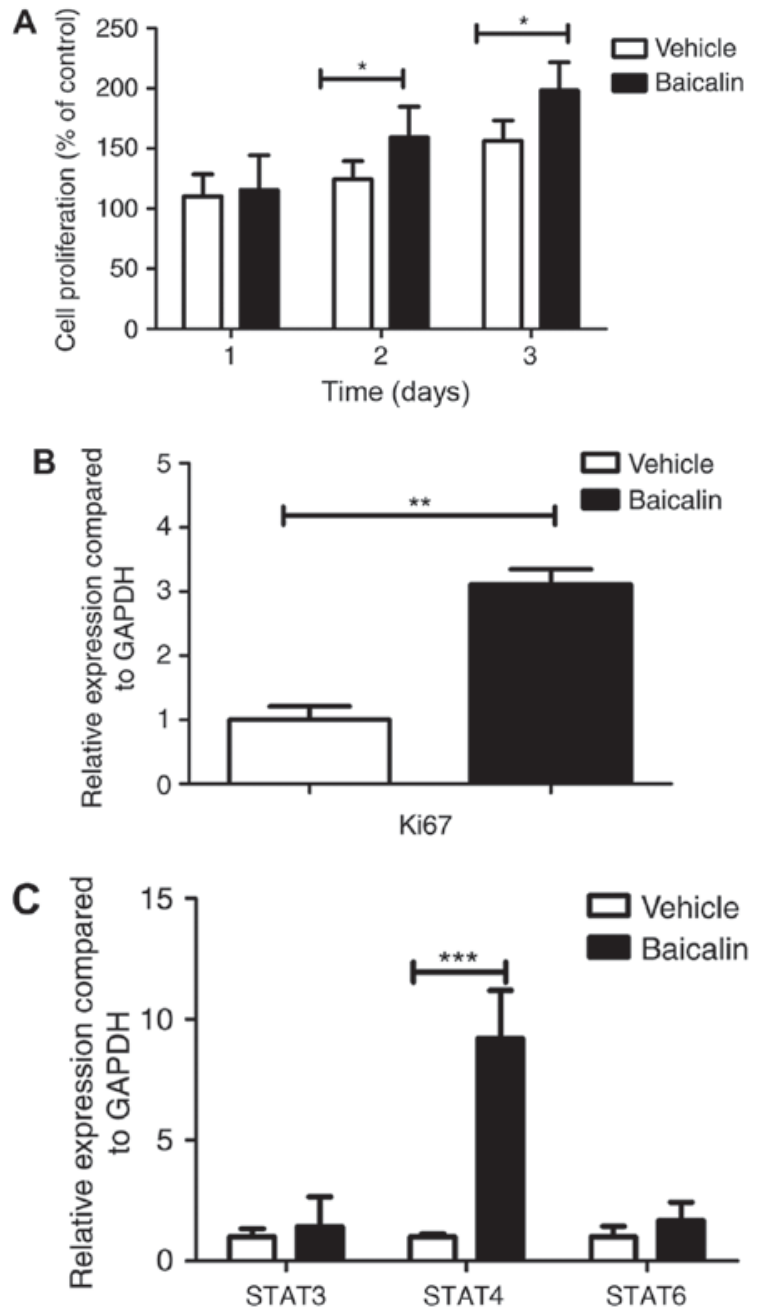

Figure 4. Baicalin regulates epithelial cell proliferation and STAT expression. (A) MTT assay of proliferation of epithelial cells isolated from the colon of DSS mice and treated with baicalin in vitro. (B) The Ki67 mRNA expression of epithelial cells isolated from colon of DSS mice and treated with baicalin in vitro. (C) The STAT3/4/6 mRNA expression of epithelial cells isolated from the colon of DSS mice and treated with baicalin in vitro. The data are presented as the mean \pm standard deviation. ${ }^{*} \mathrm{P}<0.05,{ }^{* *} \mathrm{P}<0.01$, ${ }^{* * *} \mathrm{P}<0.001$. STAT, signal transducer and activator of transcription; DSS, dextran sodium sulfate.

the apoptosis of human immunodeficiency virus-infected CEM cells (19). Furthermore, the antitumor effects of baicalein and baicalin on human hepatoma cell lines have been reported (20).

Animal models of autoimmune arthritis and colitis have proven to be valuable research tools for the study of the pathogenic mechanisms of these autoimmune diseases, in addition to the testing of novel therapeutics. Firstly, the present study identified that baicalein was effective in the murine RA model induced by type II collagen. CIA has been the most widely used model of RA; it shares several pathological features with RA, and collagen is an important protein in cartilage, the target tissue in RA $(21,22)$. Additionally, of the antigen-defined models that are based on cartilage proteins, CIA has the shortest duration between immunization and disease manifestation. In the present study, treatment with baicalein in vivo reduced the arthritis score and attenuated the expression of inflammatory cytokines in the serum. This was due to the in vitro regulation of $\mathrm{T}$ cell proliferation via the JAK-STAT signaling pathway; in particular, it suppressed the expression of STAT3 and STAT4. In another experiment, it was demonstrated that the Scutellariae radix component baicalin alleviated disease severity in the mouse colitis model induced by DSS. Inflammatory bowel disease (IBD) is an autoimmune disease characterized by chronic, uncontrolled inflammation in the intestinal mucosa, affecting millions of people worldwide. The DSS-challenged mice exhibited stable symptoms of IBD, with weight loss, shorter colon length, rectal bleeding and loose stools (23). However, the control mice exhibited more severe disease compared with baicalin-treated animals, which presented with reduced weight loss and increased colon length. Furthermore, proinflammatory responses were suppressed in the colon in baicalin-treated mice. In contrast to the inhibition of $\mathrm{T}$ cell proliferation in the RA model induced by baicalein, the other Scutellariae radix component baicalin was able to promote epithelial proliferation by amplifying STAT4 gene expression and the downstream pathway. The JAK-STAT pathway is a direct signal transduction pathway in the nucleus: The extracellular signaling proteins, including cytokines (for example, IFN- $\gamma$ and interleukins) and growth factors, interact with transmembrane receptors at the cell surface in order to monitor the extracellular environment, JAK family gene expression is activated and the substrate STAT proteins are phosphorylated $(24,25)$. Different STAT subtypes are involved in cell proliferation, differentiation and transformation (26-28). The results of the present study demonstrated that the natural compounds of Scutellariae radix were able to directly regulate the expression of STAT genes in the JAK-STAT pathway. In conclusion, the two components of Scutellariae radix, baicalein and baicalin, exhibited therapeutic effects in two types of autoimmune disease. The two compounds were able to modulate the proliferation of the target cells in arthritis and colitis through various STAT subtypes in the JAK-STAT pathway (baicalein, STAT3/4; baicalin, STAT4).

\section{Acknowledgements}

Not applicable.

\section{Funding}

The present study was supported by the National Natural Science Foundation of China (grant no. 81401230).

\section{Availability of data and materials}

The datasets used and/or analyzed during the current study are available from the corresponding author on reasonable request.

\section{Authors' contributions}

JX and ZG designed and performed the experiments, analyzed the data, and wrote the manuscript. JLiu, GY, MS, JLi and XX performed experiments and data analysis.

\section{Ethics approval and consent to participate}

All animal procedures were performed in strict accordance with the recommendations in the Guide for the Care and Use of the Animal Biosafety Level 3 Laboratory of Wuhan 
University (Wuhan, China). The protocol was approved by the Committee on the Ethics of Animal Experiments of Wuhan University (permit no. SCXK 2008-0004).

\section{Consent for publication}

Not applicable.

\section{Competing interests}

The authors declare that they have no competing interests.

\section{References}

1. Tobón GJ, Youinou P and Saraux A: The environment, geo-epidemiology, and autoimmune disease: Rheumatoid arthritis. J Autoimmun 35: 10-14, 2010.

2. Kragstrup TW, Jalilian B, Keller KK, Zhang X, Laustsen JK, Stengaard-Pedersen K, Hetland ML, Hørslev-Petersen K, Junker P, Østergaard M, et al: Changes in soluble CD18 in murine autoimmune arthritis and rheumatoid arthritis reflect disease establishment and treatment response. PLoS One 11: e0148486, 2016

3. Pan TL, Wang PW, Huang CH, Leu YL, Wu TH, Wu YR and You J: Herbal formula, Scutellariae radix and Rhei rhizoma attenuate dimethylnitrosamine-induced liver fibrosis in a rat model. Sci Rep 5: 11734, 2015.

4. Chen CY, Chen JX, Li W, Li H and Yang B: Comparative chemical and statistical analysis of cultivated and wild Radix Scutellariae. Am J Chin Med 39: 1029-1041, 2011.

5. Ikemoto S, Sugimura K, Yoshida N, Yasumoto R, Wada S, Yamamoto K and Kishimoto T: Antitumor effects of Scutellariae radix and its components baicalein, baicalin, and wogonin on bladder cancer cell lines. Urology 55: 951-955, 2000.

6. Li HL, Zhang S, Wang Y, Liang RR, Li J, An P, Wang ZM, Yang J and Li ZF: Baicalein induces apoptosis via a mitochondrial-dependent caspase activation pathway in T24 bladder cancer cells. Mol Med Rep 7: 266-270, 2013.

7. Shieh DE, Liu LT and Lin CC: Antioxidant and free radical scavenging effects of baicalein, baicalin and wogonin. Anticancer Res 20: 2861-2865, 2000

8. Xu J, Zhang Y, Xiao Y, Ma S, Liu Q, Dang S, Jin M, Shi Y, Wan B and Zhang Y: Inhibition of 12/15-lipoxygenase by baicalein induces microglia PPAR $\beta / \delta$ : A potential therapeutic role for CNS autoimmune disease. Cell Death Dis 4: e569, 2013.

9. Suk K, Lee H, Kang SS, Cho GJ and Choi WS: Flavonoid baicalein attenuates activation-induced cell death of brain microglia. J Pharmacol Exp Ther 305: 638-645, 2003.

10. Chen CJ, Raung SL, Liao SL and Chen SY: Inhibition of inducible nitric oxide synthase expression by baicalein in endotoxin/cytokine-stimulated microglia. Biochem Pharmacol 67: 957-965, 2004

11. Zhang Y, Shan L, Hua Y, Wang D, Zeng H, Liu R, Zhang W and $\mathrm{Hu} \mathrm{Z}$ : Baicalein selectively induces apoptosis in activated lymphocytes and ameliorates concanavalin a-induced hepatitis in mice. PLoS One 8: e69592, 2013.

12. Krakauer T, Li BQ and Young HA: The flavonoid baicalin inhibits superantigen-induced inflammatory cytokines and chemokines. FEBS Lett 500: 52-55, 2001.
13. Li BQ, Fu T, Gong WH, Dunlop N, Kung H, Yan Y, Kang J and Wang JM: The flavonoid baicalin exhibits anti-inflammatory activity by binding to chemokines. Immunopharmacology 49: 295-306, 2000

14. Livak KJ and Schmittgen TD: Analysis of relative gene expression data using real-time quantitative PCR and the 2(-Delta Delta C(T)) method. Methods 25: 402-408, 2001.

15. Park CM, Reid PE, Walker DC and MacPherson BR: A simple, practical 'swiss roll' method of preparing tissues for paraffin or methacrylate embedding. J Microsc 145: 115-120, 1987.

16. $\mathrm{Xu}$ Y, Hunt $\mathrm{NH}$ and Bao S: The role of granulocyte macrophage-colony-stimulating factor in acute intestinal inflammation. Cell Res 18: 1220-1229, 2008

17. Takasuna K, Kasai Y,Kitano Y,Mori K,Kobayashi R,Hagiwara T, Kakihata K, Hirohashi M, Nomura M, Nagai E, et al: Protective effects of kampo medicines and baicalin against intestinal toxicity of a new anticancer camptothecin derivative, irinotecan hydrochloride (CPT-11), in rats. Jpn J Cancer Res 86: 978-984, 1995.

18. Lin M, Li L, Li L, Pokhrel G, Qi G, Rong R and Zhu T: The protective effect of baicalin against renal ischemia-reperfusion injury through inhibition of inflammation and apoptosis. BMC Complement Altern Med 14: 19, 2014.

19. Baylor NW, Fu T, Yan YD and Ruscetti FW: Inhibition of human T cell leukemia virus by the plant flavonoid baicalin (7-glucuronic acid, 5,6-dihydroxyflavone). J Infect Dis 165: 433-437, 1992.

20. Chang WH, Chen CH and Lu FJ: Different effects of baicalein, baicalin and wogonin on mitochondrial function, glutathione content and cell cycle progression in human hepatoma cell lines. Planta Med 68: 128-132, 2002.

21. Di Sante G, Tolusso B, Fedele AL, Gremese E, Alivernini S, Nicolò $C$, Ria F and Ferraccioli G: Collagen specific T-cell repertoire and HLA-DR alleles: Biomarkers of active refractory rheumatoid arthritis. EBioMedicine 2: 2037-2045, 2015.

22. Schultz HS, Guo L, Keller P, Fleetwood AJ, Sun M, Guo W, Ma C, Hamilton JA, Bjørkdahl O, Berchtold MW and Panina S: OSCAR-collagen signaling in monocytes plays a proinflammatory role and may contribute to the pathogenesis of rheumatoid arthritis. Eur J Immunol 46: 952-963, 2016.

23. Palone F, Vitali R, Cucchiara S, Pierdomenico M, Negroni A, Aloi M, Nuti F, Felice C, Armuzzi A and Stronati L: Role of HMGB1 as a suitable biomarker of subclinical intestinal inflammation and mucosal healing in patients with inflammatory bowel disease. Inflamm Bowel Dis 20: 1448-1457, 2014.

24. Liongue C, O'Sullivan LA, Trengove MC and Ward AC: Evolution of JAK-STAT pathway components: Mechanisms and role in immune system development. PLoS One 7: e32777, 2012.

25. Abell $\mathrm{K}$ and Watson CJ: The Jak/Stat pathway: A novel way to regulate PI3K activity. Cell Cycle 4: 897-900, 2005.

26. Zoranovic T, Grmai L and Bach EA: Regulation of proliferation, cell competition, and cellular growth by the Drosophila JAK-STAT pathway. JAKSTAT 2: e25408, 2013.

27. Kowshik J, Baba AB, Giri H, Deepak Reddy G, Dixit M and Nagini S: Astaxanthin inhibits JAK/STAT-3 signaling to abrogate cell proliferation, invasion and angiogenesis in a hamster model of oral cancer. PLoS One 9: e109114, 2014.

28. Katsuyama T, Comoglio F, Seimiya M, Cabuy E and Paro R: During Drosophila disc regeneration, JAK/STAT coordinates cell proliferation with Dilp8-mediated developmental delay. Proc Natl Acad Sci USA 112: E2327-E2336, 2015. 